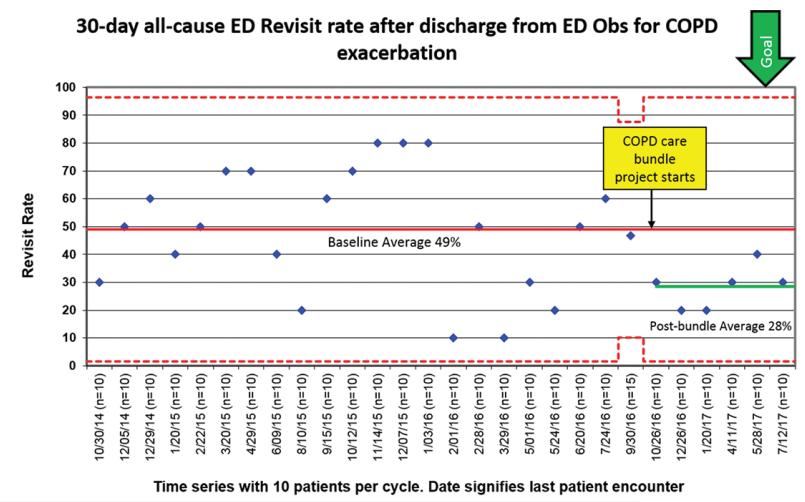

- Percentage of patients with 30-day revisit — Average 30-day Revisit Rate -----Control Limits —— Post-bundle Average COPD: chronic obstructive pulmonary disease

Abstract 1005 Figure 3 Outcome measure: 30 day all-cause ED revisit rate. COPD: chronic obstructive pulmonary disease

Objectives Achieve 90\% reliable implementation of COPD care-bundle among eCOPD patients discharged from ED-Obs by April 2017. The overall goal is to reduce 30 day all-cause ED revisits for eCOPD patients discharged from ED-Obs from the baseline rate of $49 \%$.

Methods Setting: An 800-bed academic hospital with 700 eCOPD ED encounters/year, out of which 20\% triage to EDObs. All patients triaged to ED-Obs with eCOPD diagnosis were included. A five-element COPD bundle designed to mitigate system-level failures, was adopted from in-patient setting. The bundle components were: appropriate inhaler regimen, 30 day inhaler supply, personalised inhaler education, standardised discharge instructions and follow-up in 15 days. A multidisciplinary team was formed to support bundle implementation within the 24 hours ED-Obs stay using Model for Improvement. Bundle component adherence and 30 day ED revisit rates were monitored using SPC p-charts. Hospitalisation rate from ED-Obs was used as balancing measure.

Results The patient characteristics were similar in baseline and post-bundle period. Multiple PDSA cycles were performed to achieve a final process (Figure 1). The adherence to COPD care bundle components has maintained $>90 \%$ from 4/2017$8 / 2017$ (Figure 2). The 30 day all-cause $\mathrm{ED}$ revisit rate reduced from $49 \%$ to $28 \%$ with a pending system-shift on SPC (Figure 3). Hospitalisation rate remained unchanged.

COPD: chronic obstructive pulmonary disease, ED: emergency department, Obs: observation, $\mathrm{d} / \mathrm{c}$; discharge, dispo: disposition, Rx: prescription, HER: electronic health record, INH: inhalers, PCP: primary care provider

RT: respiratory therapists, MLP: mid-level provider, EMR: electronic health record

COPD: chronic obstructive pulmonary disease

Conclusions Improving care at discharge transition of eCOPD patients from ED-Obs to home through reliable adherence to COPD care-bundle reduces 30 day all-cause ED revisits.

\section{A QUALITY IMPROVEMENT (QI) COLLABORATIVE TO IMPROVE INFLUENZA IMMUNISATION RATES FOR DISEASE-SPECIFIC PAEDIATRIC POPULATIONS}

Haley S Friedler, Patricia Meleedy-Rey, Jessica B Kerr, Tracy Liwen Chen, Maurice W Melchiono, Patricia Mantell, Mary E Poyner Reed, Taruna Banerjee. Boston Children's Hospital, US

\subsection{6/bmjoq-2017-IHI.22}

Background Influenza outbreaks are a major public health concern yet the majority of work to improve immunisation rates has occurred within primary care settings. Children and patients with medical complexity are particularly at risk for complications due to influenza. Emphasis on immunising all patients against the flu, including patients seen in specialty clinics, is essential to help prevent the acquisition and spread of the influenza virus.

Objectives To improve flu immunisation rates for paediatric patients with chronic diseases across multiple medical divisions representing unique patient populations.

Methods Over a five year period we oversaw a multi-disciplinary, nursing-focused QI collaborative including primary care and specialty clinics at a freestanding children's hospital. The QI collaborative included monthly meetings with divisional champions, nursing leadership, pharmacy, and marketing for data review and synergistic learning. Key improvement initiatives included the development of standardised division-specific processes, implementation of a web-based dashboard for realtime data feedback, phone calls to patients and families to encourage and document flu immunizations, and a hospitalwide marketing campaign focused on patient and provider education. Five divisions followed the same disease-specific population, tracking data monthly, over the five year period.

Results From the 2013 to the 2017 flu season, immunisation rates increased significantly $(\mathrm{p}=0.0270)$. Populations included patients with sickle cell disease $(58 \%$ to $84 \%)$, inflammatory bowel disease (35\% to 65\%), cystic fibrosis (79\% to $89 \%)$, diabetes $(20 \%$ to $70 \%)$, and patients on dialysis $(89 \%$ to 100\%).

Conclusions The creation of a multidisciplinary, nursingfocused collaborative was associated with significant improvements in influenza immunisation rates in specialty populations.

\section{THE USE OF SENSORY MODALITIES TO POSITIVELY IMPACT BEHAVIOURS OF ADOLESCENTS WITH SENSORY MODULATION DISORDERS WITHIN AN INPATIENT PSYCHIATRIC SETTING}

${ }^{1}$ Cristina Ranches, ${ }^{1}$ Susana Wyatt, ${ }^{2}$ Liz Budek. 'LAC+USC Medical Centre, Augustus F. Hawkins, US: ${ }^{2}$ LAC+USC Medical Centre, US

\subsection{6/bmjoq-2017-IHI.23}

Background Adolescents with sensory modulation disorders (SMD) have a high incidence of acting out inappropriately due to difficulty focusing and self-regulating. Use of sensory 